\title{
Jean-Luc Sochacki
}

Centre d'Études et de Recherches en Sciences de l'Éducation (CERSE)

Université de Caen, France

http://dx.doi.org/10.18778/8088-896-8.10

\section{LA MINORITÉ POLONAISE OU LA PRÉHISTOIRE DES ENSEIGNEMENTS LANGUES ET CULTURES D'ORIGINES EN FRANCE (1919-1939)}

En 1919, France et Pologne signent une convention d'immigration/émigration visant à faciliter l'envoi de travailleurs polonais vers une France à reconstruire. Dans ce texte, rien n'était indiqué sur la scolarisation des enfants polonais. Si la question de l'obligation scolaire restera floue de 1919 à $1936^{1}$, il en sera de même pour la mise en place de classes en langue polonaise dans le système scolaire français. Employeurs français, autorités polonaises et ouvriers polonais vont alors instaurer des « classes polonaises ». Face à cela, plusieurs circulaires gouvernementales sont publiées au cours des années 1920, faisant alors des entorses au principe de non-différentiation de l'élève au sein de l'école Républicaine. C'est ce que nous pouvons nommer la « préhistoire » des enseignements Langues et cultures d'origines telles qu'ils seront pensés en France au XX' siècle.

À travers cette question des enseignements polonais en France entre 1919 et 1939, il est intéressant de voir comment la minorité polonaise va être à l'origine et va influencer (de manière durable) ces enseignements Langues et cultures d'origines en France (toujours au centre des débats à l'heure actuelle).

Dans une première partie, nous nous intéresserons à la convention de 1919 entre France et Pologne et à l'impensé scolaire. Dans une seconde partie, nous verrons comment, de manière concrète, ce courant migratoire va peser et influencer le système scolaire français. Enfin, nous étudierons un cas local de classe polonaise dans un village de Normandie largement 'polonisé' : Potigny.

1 Il faut attendre la loi du Front populaire date du 9 août 1936 pour mettre définitivement un terme à cette ambigüité en stipulant « l'instruction primaire obligatoire pour les enfants des deux sexes, français et étrangers, de six ans à quatorze ans révolus ». En ce qui concerne les enfants Polonais, le protocole né de la conférence franco-polonaise du 27 avril 1924 indiquait toutefois que l'obligation scolaire proscrite par la loi du 28 mars 1882 s'imposait aux enfants d'ouvriers polonais comme à tous les enfants qui résident sur le territoire français. 


\section{La convention de 1919 ou le début de la Stara emigracja}

Avant la Première guerre mondiale, l'industrie française fait appel aux Polonais. En effet, des petites colonies de Polonais étaient déjà présentes dans le Nord de la France. Des échanges de courriers conservés aux Archives départementales du Calvados font état de 150 Polonais à Aniche, 30 à Anzin, 30 à Marles, quelques-uns à Lens et Béthune employés entre autre à la Compagnie des mines de Vicoignes et de Noeux-les-mines. Dans le Calvados, ces archives font état de contacts entre les mines de Soumont et les services du Prince Witold Czartoryski dans le but de recruter des ouvriers, ce qui avant la Première guerre ne se fera pas (la piste italienne étant alors privilégiée). Par contre, après ce premier conflit mondial, la France va faire massivement appel aux Polonais.

\section{Un cruel besoin de main-d'ouvre et le rôle du Comité central des houillères de France (CCHF)}

C'est dans l'urgence et l'optique de la reconstruction du pays après 1918 que France et Pologne vont mettre en œuvre une convention visant à l'envoi de travailleurs Polonais vers la France, pays qui manque alors cruellement de maind'œuvre. Des 207000 mineurs en activité en décembre 1918, il n'en restait plus que 163000 au $1^{\text {er }}$ juillet 1919. Il faut dire que cette immense perte d'hommes aux combats de la guerre 1914-1918 (plus d'un million trois cent mille morts et plus de quatre millions de blessés) se trouvait conjuguée à une situation démographique peu florissante avant-guerre. Tout au long du XIX ${ }^{\mathrm{e}}$ siècle, la France avait connu une forte baisse du taux de natalité et plusieurs années où le taux de mortalité l'emportait même sur le taux de natalité (ce fut, par exemple, le cas en 1870-1871 à cause de la guerre franco-allemande).

De son côté, la Pologne connaissait alors toutes les difficultés d'un état qui renaissait une nouvelle fois de ses cendres à la suite du Traité de Versailles de 1919. L'instabilité politique régnait, les difficultés économiques étaient conséquentes tant dans le secteur industriel (important retard) que dans le secteur agricole (phénomène des « propriétés naines », c'est-à-dire moins de 2 ha, et surpeuplement des campagnes). Comme le note Janine Ponty, la population rurale passait de 4,8 millions d'habitants en 1860 à 13 millions en 1913. Ponty relève qu'au « surpeuplement des campagnes, s'ajoute l'engorgement des villes qui, malgré l'existence d'activités industrielles, ne peuvent fournir du travail à tout le monde $\gg^{2}$. Dans les campagnes, « la surpopulation rurale oblige des familles très nombreuses à vivre sur de minuscules parcelles qui ne fournissent pas du travail à tout le monde $\gg^{3}$.

2 J. Ponty, Polonais méconnus. Histoire des travailleurs immigrés en France dans l'entredeux-guerres, Paris, Publications de la Sorbonne, 2005, p. 42.

3 Ibidem. 
Dans ce contexte, le départ de nombre d'habitants vers la France représentait une solution surtout que les U.S.A, terre d'accueil de plus de deux millions de Polonais au XIX ${ }^{e}$ siècle, mettaient en place une politique de quotas visant à une forte réduction du nombre d'immigrés, comme le Literacy test instauré en février 1917, The emergency quota act de 1921 et The Johnson-Reed Act de 1924.

Aussi, Janine Ponty explique que le Comité Central des Houillères de France (C.C.H.F) qui est à l'origine, intermédiaire et interlocuteur privilégié entre les deux États va alors refuser la mécanisation et continuer à privilégier l'humain. $\mathrm{Si}$ la mécanisation pouvait être une solution concernant le travail d'extraction dans les mines, elle ne se révélait pas être d'une grande utilité car la majeure partie du travail consistait alors dans la remise en état des puits détruits et ennoyés (travaux de boisage, déblayage...) Car il ne faut pas s'y tromper, bien plus que le gouvernement français, c'est les dirigeants du Comité central des houillères de France qui menaient les négociations. C'était eux, les grands bénéficiaires de cette convention. Cette mainmise des industriels sur les recrutements de travailleurs étrangers suscitait d'ailleurs la crainte et l'inquiétude des politiques. Certains voyaient d'un mauvais œil ces arrivées massives de travailleurs étrangers qui échappaient globalement au contrôle de l'État. C'était le cas du député radical de Lyon, Charles Lambert, qui affirmait :

Le recrutement des ouvriers étrangers étant principalement l'œuvre des grandes organisations patronales dont l'intérêt est d'avoir une main-d'œuvre qui sera d'autant plus malléable qu'elle restera en dehors des organisations syndicales ouvrières françaises, de considérables groupements homogènes d'ouvriers appartenant à un même pays, comprenant surtout des Polonais et des Italiens, se sont formés dans certaines régions où ils ont constitué de véritables entités nationales. C'est ainsi que dans le département du Nord qui compte officiellement 233026 étrangers sur une population de 1969159 habitants, il y a des villages entiers de Polonais, venus avec leurs femmes et leurs enfants, suivis de leurs prêtres et de leurs instituteurs, et qui, ainsi, pendant longtemps, seront rebelles à toute assimilation ${ }^{4}$.

\section{Une convention improvisée ?}

Concernant cette convention, Janine Ponty note :

À en juger par l'indigence des sources, on croirait que la convention du 3 septembre 1919 est née de génération spontanée : presque rien avant la signature ni aussitôt après, mais le texte lui-même en bonne place dans les dossiers d'Archives. Il n'est pas impossible que ce silence soit le reflet de la réalité, que les séances de travail aient

${ }^{4}$ C. Lambert, La France et les étrangers. Dépopulation. Immigration. Naturalisation, Paris, Librairie Delagrave, 1928, p. 63. 
été d'autant moins nombreuses que la délégation polonaise accepte très vite ce que la France lui propose, faute d'oser faire autrement tant pour ne pas vexer la nation amie que parce qu'il est urgent d'aboutir'.

Le rapport de force entre les deux pays était clairement déséquilibré, la situation économique en Pologne était telle, que dans ces 'négociations', elle était littéralement acculée.

Dans cette convention, il est important de noter que plusieurs points sont oubliés comme les questions concernant le regroupement familial. Un oubli nous intéresse tout particulièrement : rien dans ce texte n'était indiqué concernant la scolarisation des enfants polonais de moins de treize ans qui résidaient alors en France.

\section{Les conséquences démographiques}

Le nombre de Polonais en France entre les deux guerres va rapidement augmenter et devenir conséquent. En 1921, les conséquences de la convention signée deux ans plus tôt ne sont pas encore visibles, ils sont 46000 loin derrière les Italiens (451 000), les Espagnols (259 000) ou les Belges (349 000). En 1926, les Polonais sont 309 000, quatrième nationalité étrangère présente dans l'hexagone, toujours loin derrière les Italiens dont le nombre a aussi sensiblement augmenté (750 000 dont beaucoup fuient la dictature de Mussolini), les Belges (327 000) et les Espagnols (323 000). En 1931, malgré la Crise de 1929, les Polonais représentent la seconde nationalité étrangère (508 000) derrière les Italiens (808 000). Il en sera de même en 1936 (423000 Polonais, 721000 Italiens). Pour faire face à cette vague migratoire polonaise, il existait en 1924, huit circonscriptions consulaires : Lille, Paris, Strasbourg, Lyon, Bordeaux, Nice, Marseille et le Havre.

\section{Les classes polonaises de la Stara emigracja ou l'influence de la minorité polonaise dans le système scolaire français}

\section{Les cours de polonais ou l'enjeu essentiel du maintien de la polonité}

Comme nous l'avons vu, la Convention d'immigration/émigration de 1919 entre France et Pologne ne stipulait rien concernant l'éducation des enfants polonais en France. Dans l'optique d'un retour prochain et probable au pays, il convenait de maintenir la polonité et cela passait par un apprentissage de la langue d'origine mais aussi de l'histoire et de la géographie de la Pologne. Si les Polonais envoient leurs enfants à l'école publique française, il est ici intéressant de noter

\footnotetext{
5 J. Ponty, op. cit., p. 45.
} 
qu'à l'époque ils ne souhaitent pas s'intégrer complètement. Nous ne pouvons évoquer l'immigration polonaise de l'entre-deux-guerres sans aborder l'idée du provisoire très présente dans les esprits et étroitement liée à l'idée de maintenir cette polonité vivante en utilisant, entre autre, des structures scolaires ${ }^{6}$. Effectivement, pour les autorités des deux pays comme pour les émigrés/immigrés polonais, ce courant migratoire était pensé comme provisoire. Quand les conflits (Première guerre mondiale et guerre russo-polonaise (février 1919-mars 1921) seront terminés et leurs conséquences atténuées, quand la situation économique du pays sera rétablie alors les Polonais pourront retrouver leur pays.

Le maintien d'un fort sentiment de polonité était voulu, désiré et encouragé par les autorités polonaises qui impliquaient des résistances au processus d'intégration incarnées entre autre dans les associations (culturelles, religieuses, sportives...) et les classes pour enfants en langue polonaise. Jules Chœur évoquait d'ailleurs chez les Polonais « une vie communautaire d'une intensité exceptionnelle que l'on retrouve chez aucune autre population étrangère en France $\gg^{7}$.

Ainsi, Janine Ponty note que des cas de cours de polonais hors de tout contrôle de l'État en France sont mis en place dès le début des années 1920. Par exemple, à Knutange (complexe sidérurgique proche de Thionville) un cours est instauré en 1922. La même année, les mines d'Ostricourt prennent en charge ce type d'enseignement pour les enfants d'ouvriers immigrés (mise à disposition de locaux et recrutement de deux moniteurs). En 1923, les Polonais de Ronchamp (Haute-Saône) « demandent et obtiennent un arrêté ministériel leur permettant d'ouvrir un cours de langue pour leurs enfants $\gg^{8}$. Dès le début des années 1920 et suite à l'impensé de la question scolaire dans la convention de 1919, l'État de France doit faire face à cette mise en place de cours en langue d'origine pour enfants polonais.

\section{La question scolaire polonaise en France ou le résultat d'un compromis}

De son côté, l'État polonais se penche aussi sur ce problème. En avril 1924, la question de l'ouverture de cours de langue et culture d'origine pour les enfants d'immigrés est soulevée lors de la Conférence franco polonaise qui se tient

${ }^{6}$ Cela avait déjà été le cas au XIX ${ }^{\mathrm{e}}$ siècle avec l'instauration en 1842 de l'École nationale polonaise de Paris (Szkoła Narodowa Polska w Paryżu) plus connue sous le nom d'École polonaise des Batignolles (nom lié au quartier dans lequel cette institution s'installe en 1844).

${ }^{7}$ J. Chœur, Évolution démographique de la région de Douai depuis l'exploitation du bassin minier. In : Revue du Nord, t. 36, n 143, juillet-septembre 1954.

8 J. Ponty, op. cit., p. 158. 
à Paris. Les autorités françaises refusent de légiférer et d'institutionnaliser ces enseignements mais pour ne pas décevoir cet important partenaire polonais, le Comité central des houillères françaises par l'intermédiaire d'Henri de Peyerimhoff rédige une lettre où il invite les employeurs à mettre en œuvre ce type de leçon selon deux façons :

a) soit dans les écoles publiques, en supportant les frais de l'enseignement complémentaire qui pourrait être institué.

b) soit, lorsque l'effectif des enfants polonais le justifiera, en ouvrant, au fur et à mesure des besoins, des écoles privées où sera assuré, sous la surveillance des autorités académiques françaises et dans le cadre du programme scolaire normal, le même enseignement complété, s'il y a lieu, pour les jeunes enfants qui n'auraient pas encore les notions suffisantes de la langue française, par l'enseignement d'autres matières en polonais'.

En France, la lettre ne paraît pas au journal officiel et reste confidentielle. En revanche, Janine Ponty rapporte qu'en Pologne, « Varsovie l'insère, traduite en Polonais dans le recueil des accords polono-français relatifs à l'émigration $\gg^{10}$ (Zestawienia tekstów polsko francuskich umów emigracyjnych) et la considère comme une charte de l'enseignement polonais en France.

À travers le financement par le Comité Central des Houillères de France, de cours de langues étrangères aux enfants d'immigrés dans les écoles publiques, il s'agit d'une entorse au principe de non-différentiation au sein de l'école Républicaine et à l'idée d'un élève « neutre ». L'origine nationale de l'élève est alors prise en considération. Ainsi, selon Janine Ponty, en octobre 1924, c'était 9588 jeunes polonais qui fréquentaient ces enseignements ( 5755 dans les établissements des houillères et 3833 à l'école laïque).

\section{Les premiers textes officiels}

À la suite de ces mises en place de cours de langue pour enfants d'étrangers, le Ministère français de l'Instruction prend ses responsabilités concernant cette question et publie plusieurs circulaires. Entre autre celle du 30 juin 1924 qui indique la possibilité d'accueillir des « moniteurs » qui apprendraient la langue maternelle des enfants immigrés puis celle du 21 décembre 1925 qui rappelle la possibilité d'assurer l'enseignement d'une langue étrangère dans les écoles publiques après les heures de classes régulières et dans les écoles privées, la possibilité d'intervention en langue étrangère de maîtres étrangers sous l'autorité de maîtres français pendant le temps scolaire (dans les limites de la moitié du temps). Enfin suivront celles du 13 décembre 1927 et du 28 mars 1929.

9 Cité par J. Ponty, ibidem, p. 156.

10 Ibidem, p. 157. 
Le 12 juillet 1939, une circulaire de synthèse (rappel des circulaires de 1925, 1927 et 1929) concernant les moniteurs étrangers et conditions d'ouverture des cours de langues étrangères était publiée. C'est ce texte qui fut très largement repris dans la circulaire du 30 mars 1976 qui permet l'utilisation des locaux scolaires par les enseignants étrangers dans le cadre des classes d'enseignement en langue et culture d'origine (ELCO).

\section{III. Étude de cas, un exemple local : Potigny (France - Calvados)}

\section{La population polonaise de Potigny}

Nous évoquerons ici le village de Potigny (au sud de Caen) voisin de Soumont-Saint-Quentin qui possédait une mine de fer et qui fut à l'origine de la venue de ces Polonais. Au recensement de 1926, les Polonais étaient 383 sur une population de 1731 habitants et c'est à partir de ces années que Potigny va devenir la « Petite Varsovie » avec ses associations, son prêtre et son « moniteur » nommé par le Consulat de Pologne et choisi par la direction de la mine de Soumont. En 1931, la communauté polonaise est la plus nombreuse et représente $27 \%$ des étrangers devant les Italiens (18,5\%). Il en va encore ainsi en 1936 où la colonie polonaise représente $28,3 \%$ de la population étrangère tandis que les Italiens sont à la deuxième position avec $17,2 \%$. Cette même année, Potigny se classe à la onzième position des vingt-quatre villages qui comptent plus d'habitants Polonais que de Français (1373 sur une population de 2742 habitants).

\section{Une volonté politique}

L’année 1926 est marquée par l'arrivée de Polonais à Potigny appelés pour travailler dans les mines de fer de la commune voisine de Soumont (Société des mines de Soumont). Dès cette année, une communauté forte et bien structurée se met en place encouragée entre autre par les autorités consulaires polonaises. Le fonds archivistique de la Société des mines de Soumont conservé aux Archives départementales du Calvados montre bien ce fait. Le Consulat apporte un soutien sans faille à cette politique qui vise à entretenir l'amour de la patrie dans l'espoir d'un retour des polonais au pays. Les Polonais ne comptent certainement pas passer leurs vies en France. Le maintien du sentiment national, de la polonité s'avère donc essentiel. Les autorités polonaises en France prônaient avant tout une vision nationaliste et visaient à maintenir les Polonais (entre autre, dans les cités ouvrières) dans une atmosphère empreinte de polonité en développant les manifestations et en perpétuant la langue au moyen des classes polonaises. Il s'agit alors de garder ce lien fort entre le pays que l'on vient de quitter et qu'on ne saurait tarder à retrouver. 
À Potigny, cette entreprise est facilitée par la direction de la mine qui mettait tout en œuvre pour que les nouveaux venus se sentent «comme chez eux ». Si aucune politique préalable concernant l'installation d'une communauté étrangère n'était pensée ou anticipée dans l'esprit de la direction, cette dernière acceptait les (nombreuses) requêtes émanant de la communauté Polonaise afin d'éviter les conflits avec cet important contingent de travailleurs et tenter ainsi de stabiliser cette main-d'œuvre.

\section{Français et Polonais, deux entités bien distinctes et une vie en vase clos au rythme des organisations polonaises (associations et classes polonaises)}

À Potigny, cette polonité s'affichait à travers une vie communautaire très fermée. Tout d'abord, une donnée démographique était significative de ce cloisonnement : on ne dénombrait que 5 mariages mixtes français-polonais entre 1926 et 1932. Ensuite, donnée « géographique », les Polonais habitaient à l'écart, à l'ouest du village. Enfin, donnée linguistique, ils ne parlaient pas français et ne voyaient pas l'intérêt de l'apprendre. Si les enfants fréquentent l'école française, les parents craignent la perte de la langue maternelle, c'est pourquoi nombre de petits Polonais assistaient aux cours donnés par le moniteur, qui était alors un personnage important de la communauté car il permettait d'apprendre et maintenir la langue d'origine et symbolisait le lien entre pays d'origine et pays d'accueil.

La vie communautaire fermée passait par les structures associatives. Nous pouvons évoquer les sokót (clubs de gymnastique, athlétisme...) ou les associations culturelles (comme la troupe de théâtre Le Réveil). Mais aussi par ses commerces.

Enfin, la vie communautaire des Polonais passait aussi par « l'école polonaise $\gg$.

On retrouve les premières traces de la présence d'un moniteur polonais à Potigny dès 1926. Cet enseignement était privé car il était instauré par la Direction de la compagnie des mines de Soumont à la demande des parents polonais. Il jouait pleinement ce rôle de maintien de la polonité des enfants dans l'optique d'un retour prochain au pays. D'ailleurs, lors d'une réunion publique de la communauté de Potigny en 1929, le prêtre polonais de la commune signalait que

parmi les Polonais qui ont beaucoup d'enfants à Potigny, un instituteur polonais est nécessaire, car nous risquons de perdre ces enfants de notre patrie, ils ne parlent presque plus notre langue, ils deviennent tout à fait français. Rencontrant des enfants dans Potigny en voulant les réconforter en parlant de la patrie et de religion, c'est à peine s'ils pouvaient me répondre et ne savaient même pas prononcer le mot 
dieu dans leur langue maternelle. Cela m'inquiète et je demande à l'assistance de signer une pétition pour faire venir un instituteur polonais ${ }^{11}$.

Dès la première année, la classe polonaise animée par le moniteur Stefan Wujec (rémunéré par la direction de la SMS) comptait plus de quatre-vingt enfants et les conditions pour participer à ces classes bien établies :

À Potigny dans le département du Calvados qui était du ressort du Consulat de Paris, au $1^{\text {er }}$ octobre 1926, 39 garçons et 47 filles fréquentaient les cours de polonais dans une salle spécialement bâtie dans ce but auprès de l'école communale. La compagnie des mines de fer de Soumont supportait les frais d'entretien de cette «école polonaise », elle achetait les manuels et les fournitures scolaires. Le directeur communal n'intervenait pas dans les affaires des Polonais, mais les 105 enfants devaient être inscrits à l'école française pour être admis dans les cours de polonais. C'était là un modèle idéal de ce qui pouvait être fait dans le cadre de la législation scolaire française à l'époque ${ }^{12}$.

Le moniteur apprenait l'histoire et la géographie de la Pologne ainsi que la langue. Il était salarié de la mine et enseignait dans des locaux fournis par cette dernière. Les heures de cours avaient lieu soit le matin avant ou le soir après l'école 'communale' publique. Des heures de cours étaient aussi organisées parfois le jeudi matin.

Concernant les rapports entre ces classes et l'administration scolaire locale (inspecteur d'Académie, le Rectorat...) nous n'avons que peu de traces dans les archives et les abondantes correspondances. Les seuls échanges archivés entre la Direction de la mine et le Rectorat sont datés de 1942 et 1943 lorsque le Rectorat indiquait que les cours de polonais n'étaient plus autorisés.

Le moniteur avait donc une place particulière dans cette communauté, une figure de notable et de 'leader'. Il devait répondre aux attentes de la direction de la Compagnie d'une part, du consulat de Pologne d'autre part mais aussi des autres membres de la communauté polonaise de Potigny (et entre autre, de l'autre grande figure de la communauté : le prêtre) et représentait ce fort maintien de la polonité au sein de la communauté polonaise de Potigny.

11 Archives départementales du Calvados - Fonds de la Société des Mines de Soumont (Don Billaux), Espace archives Arcelor Mital - Dossier « Affaires polonaises - installation de la communauté polonaise $\gg$, Côte 42J391.

12 «Sprawy emigracyjno-kulturalne w Normandii » dans le journal Narodowiec du 4 février 1927. 


\section{Conclusion}

C'est dans l'urgence de la reconstruction après la guerre 1914-1918 que la convention d'émigration/immigration de septembre 1919 a été signée. L'impératif économique prévalait largement cette sur les questions d'accueil. Les nombreux oublis concernant les questions relatives à l'accueil des enfants et leur scolarisation en sont l'exemple. Les industriels qui souhaitaient stabiliser leur main-d'œuvre étrangère d'un côté et les Polonais qui, d'un autre côté, étaient dans l'optique d'un retour au pays et par là-même du maintien fort de leur polonité ont institué ces « classes polonaises $\gg$ qui, suite à la lettre de Peyerimhoff d'avril 1924, vont être instaurées dans ces écoles privées (alors très souvent liées aux industries) mais vont aussi faire leur entrée à l'école publique à travers les différentes circulaires parues dans les années 1920 qui autorisaient la présence des moniteurs étrangers. Par ce fait, la minorité polonaise des années 1919-1939 en France a fortement contribué à prendre en compte l'appartenance de l'élève au sein de l'institution scolaire (faisant une sévère entorse du principe de l'élève neutre au sein de l'école de la République) et a ouvert la voie à ce qui deviendra les classes d'enseignement en langue et culture d'origine (ELCO).

\section{The Polish minority or the prehistoric period of teaching Languages and Cultures of Origin in France (1919-1939)}

In 1919, France and Poland signed a Convention on emigration/immigration in order to expedite the sending of Polish workers to France. No clause in this document provided for the schooling of Polish children. French employers and Polish workers then set up a Polish-speaking education programme.

With a view to possibly and soon returning to their homeland, maintaining Polish identity was necessary and entailed that people learn their native language, but also all about Poland's history and geography.

Faced with the creation of these "Polish classes", several government circulars were published in the 1920s to regulate these teachings and authorise foreign instructors, thus infringing the principle of non-differentiation of children educated under the French Republican school system.

When studying this issue of Polish lessons taught in France between 1919 and 1939, it is interesting to see how the Polish minority held a vital (and enduring) role in the establishment of the Native Languages and Cultures education programme in France (the ELCO, still currently at the heart of a debate).

Keywords: immigration/emigration - Polonity - Stara emigracja - Polish Minority - Languages and Cultures of Origin (French ELCO) education programme

Mots-clés : immigration/émigration - Polonité - Stara emigracja - Minorité polonaise - Enseignement langues et cultures d'origines 